\title{
Thermodynamics of the system of massive Dirac fermions in a uniform magnetic field
}

\author{
Ren-Hong Fang, ${ }^{1}$ Ren-Da Dong, ${ }^{2}$ De-Fu Hou, ${ }^{2, \text { f }}$ and Bao-Dong Sun ${ }^{1, *}$ \\ ${ }^{1}$ Key Laboratory of Particle Physics and Particle Irradiation (MOE), \\ Institute of Frontier and Interdisciplinary Science, \\ Shandong University, Qingdao, Shandong 266237, China \\ ${ }^{2}$ Institute of Particle Physics and Key Laboratory of Quark and Lepton Physics (MOS), \\ Central China Normal University, Wuhan 430079, China
}

\begin{abstract}
We construct the grand partition function of the system of massive Dirac fermions in a uniform magnetic field from Landau levels, through which all thermodynamic quantities can be obtained. Making use of the Abel-Plana formula, these thermodynamic quantities can be expanded as power series with respect to the dimensionless variable $b=2 e B / T^{2}$. The zero-field magnetic susceptibility is expanded at zero mass, and the leading order term is logarithmic. We also calculate scalar, vector current, axial vector current and energy-momentum tensor of the system through ensemble average approach. Mass correction to chiral separation effect is discussed. For massless chiral fermions, our results recover the chiral magnetic effect for rightand left-handed fermions, as well as chiral separation effect.
\end{abstract}

\footnotetext{
* Corresponding authors. houdf@mail.ccnu.edu.cn; sunbd@sdu.edu.cn
} 


\section{INTRODUCTION}

Since the invention of the Dirac equation [1] and the discovery of the positron [2], the properties of the fermion system under an electromagnetic field have been studied for years. Under the condition of strong electric field at order $1 \mathrm{MeV}^{2}$, fermion pairs can emerge from the vacuum, which is called Schwinger pair production and changes the structure of Maxwell equation in vacuum [3, 4]. For the vacuum quantum field theory, the proper time approach was developed for fermion systems interacting with an external electromagnetic field at zero temperature [5-7]. For the finite temperature fermion systems, the imaginary-time approach is extensively used to study the thermodynamics of the systems [8-10]. The effective action for the system of massive fermions in the presence of a static background magnetic field was obtained by the computational techniques of the imaginary time formalism and the proper time method [11. Wigner function is a useful tool to study the properties of the thermodynamics and hydromechanics of the fermion system not only for the background of a pure magnetic field [12 14] but also for a general electromagnetic field [15]21], which automatically combines the effects of both the finite temperature and the electromagnetic field in a covariant and gauge-invariant way. The strong magnetic field produced by the rapidly rotating compact stars may have great impact on the state of them [22 24]. Based on the theoretical approaches, the influence of the magnetic field on the phenomenology in high energy heavy ion collisions, such as chiral magnetic effect [16, 25] 32], chiral separation effect [33 35], magnetic catalysis [36 39], ect., is being widely researched nowadays. In recent years, the method of lattice calculation is used to study the magnetic properties of QCD matter [40 42].

In this article we study the thermodynamics of the system of massive Dirac fermions in a uniform magnetic field. Dirac fermion means that the wavefunction of the fermion satisfies the Dirac equation. Firstly we construct the grand partition function of the system from the Landau levels for a single massive fermion in a uniform magnetic field. This is different from the imaginary time formalism and the proper time method used in [11], where the authors calculated the effective Lagrangian of the fermion system and the vacuum term was naturally included. In this article, we have ignored the vacuum term in the grand partition function, which is equivalent to the normal ordering description of ensemble average approach [15, 43] and different from the un-normal ordering description in recent articles [13, 20, 44]. From 
the grand partition function, we can obtain all thermodynamic quantities, which can be expanded as power series of the dimensionless variable $b=2 e B / T^{2}$ making use of the AbelPlana formula. It is worth pointing out that all thermodynamic quantities are analytic at $b=0$. However, for the massless fermion case, the expansions of some thermodynamic quantities contain a singular term $\ln b^{2}$ as discussed in a recent article by some of the authors [45]. To avoid possible confusion, we point out that, in this article the singular log term does not mean the infinity of the physical quantity itself, but means the infinity of the derivative of the physical quantity with respect to the magnetic field at $B=0$. We expand the zerofield magnetic susceptibility with respect to the dimensionless mass parameter $c=m / T$, which is consistent with the result by the proper time method [40]. In the cases of high and low temperature, the results for magnetic susceptibility are qualitatively consistent with lattice calculation. The scalar, vector current, axial vector current and energy-momentum tensor of the system are calculated through ensemble average approach. For the axial vector current, we expand the coefficient of chiral separation effect at $c=0$ to arbitrary orders, and our results recover the chiral magnetic effect for right- and left-handed fermions in massless limit.

The rest of this article is organized as follows. In Sec. II] we list the Landau levels for a single massive Dirac fermion in a uniform magnetic field. In Sec. [II] all intensive quantities are expressed by the grand partition function. In Sec. [V] making use of Abel-Plana formula, we expand all intensive quantities as power series of the dimensionless variable $b=2 e B / T^{2}$. In Sec. $\mathrm{V}$, the zero-field magnetic susceptibility is expanded with respect to the dimensionless mass parameter $c=m / T$. In Sec. VI, we calculate the scalar, and energy-momentum tensor of the system through ensemble average approach. In Sec. VII] mass correction to chiral separation effect is discussed. This article is summarized in Sec. VIII.

Throughout this article we adopt natural units where $\hbar=c=k_{B}=1$. Metric tensor is $g^{\mu \nu}=\operatorname{diag}(+1,-1,-1,-1)$. Heaviside-Lorentz convention is used for electromagnetism. 


\section{LANDAU LEVELS FOR DIRAC EQUATION IN A UNIFORM MAGNETIC FIELD}

In this article, we use chiral representation for Dirac gamma matrixes, where the explicit forms of $\gamma^{\mu}$ and $\gamma^{5}$ are

$$
\gamma^{\mu}=\left(\begin{array}{cc}
0 & \sigma^{\mu} \\
\bar{\sigma}^{\mu} & 0
\end{array}\right), \quad \gamma^{5}=i \gamma^{0} \gamma^{1} \gamma^{2} \gamma^{3}=\left(\begin{array}{cc}
-1 & 0 \\
0 & 1
\end{array}\right)
$$

where $\sigma^{\mu}=(1, \boldsymbol{\sigma}), \bar{\sigma}^{\mu}=(1,-\boldsymbol{\sigma})$, and $\boldsymbol{\sigma}=\left(\sigma^{1}, \sigma^{2}, \sigma^{3}\right)$ are the three Pauli matrixes. Dirac equation for a massive fermion in a uniform magnetic field $\boldsymbol{B}=B \boldsymbol{e}_{z}$ is

$$
i \frac{\partial \psi}{\partial t}=\left(i \boldsymbol{\alpha} \cdot \boldsymbol{D}+\gamma^{0} m\right) \psi
$$

where $\boldsymbol{\alpha}=\gamma^{0} \boldsymbol{\gamma}, \boldsymbol{D}=\left(-\partial_{x},-\partial_{y}+i e B x,-\partial_{z}\right)$, and $e, m$ are the electric charge and mass of the fermion. We chose $A^{\mu}=(0,0, B x, 0)$ for the gauge potential. For the sake of simplicity in following calculations, we will set $e B>0$ throughout this article. The range $e B>0$ can be analytically continued to the range $e B<0$ for all results in this article.

The solutions of the energy eigenvalue equation $\left(i \boldsymbol{\alpha} \cdot \boldsymbol{D}+\gamma^{0} m\right) \psi=E \psi$ give a series of Landau levels and eigenfunctions as follows,

$$
\begin{aligned}
& n=0, \quad E=\lambda E_{0}\left(k_{z}\right), \quad \psi_{0 \lambda}\left(k_{y}, k_{z} ; \boldsymbol{x}\right)=c_{0 \lambda}\left(\begin{array}{c}
\varphi_{0}(\xi) \\
0 \\
F_{0 \lambda} \varphi_{0}(\xi) \\
0
\end{array}\right) \frac{1}{L} e^{i\left(y k_{y}+z k_{z}\right)} \\
& n>0, \quad E=\lambda E_{n}\left(k_{z}\right), \quad \psi_{n \lambda s}\left(k_{y}, k_{z} ; \boldsymbol{x}\right)=c_{n \lambda s}\left(\begin{array}{c}
\left(\begin{array}{c}
\varphi_{n}(\xi) \\
i K_{n s} \varphi_{n-1}(\xi)
\end{array}\right) \\
F_{n \lambda s}\left(\begin{array}{c}
\varphi_{n}(\xi) \\
i K_{n s} \varphi_{n-1}(\xi)
\end{array}\right)
\end{array}\right) \frac{1}{L} e^{i\left(y k_{y}+z k_{z}\right)}
\end{aligned}
$$

where $\lambda= \pm 1, s= \pm 1, \xi=\sqrt{e B} x-k_{y} / \sqrt{e B}, E_{n}\left(k_{z}\right)=\sqrt{m^{2}+k_{z}^{2}+2 n e B}, \varphi_{n}(\xi)$ is the $n$-th harmonic oscillator function along $x$-axis satisfying $\int_{-\infty}^{\infty} d \xi \varphi_{n}(\xi) \varphi_{m}(\xi)=\sqrt{e B} \delta_{n m}$, and the coefficients $K_{n s}\left(k_{z}\right), F_{0 \lambda}\left(k_{z}\right), F_{n \lambda s}\left(k_{z}\right), c_{0 \lambda}, c_{n \lambda s}$ are defined as

$$
K_{n s}\left(k_{z}\right)=\frac{k_{z}+s \sqrt{k_{z}^{2}+2 n e B}}{\sqrt{2 n e B}},
$$




$$
\begin{gathered}
F_{0 \lambda}\left(k_{z}\right)=\frac{\lambda E_{0}+k_{z}}{m}, \quad F_{n \lambda s}\left(k_{z}\right)=\frac{\lambda E_{n}-s \sqrt{k_{z}^{2}+2 n e B}}{m}, \\
\left|c_{0 \lambda}\right|^{2}=\frac{1}{1+F_{0 \lambda}^{2}}, \quad\left|c_{n \lambda s}\right|^{2}=\frac{1}{\left(1+F_{n \lambda s}^{2}\right)\left(1+K_{n s}^{2}\right)} .
\end{gathered}
$$

The eigenfunctions are set up in a cube with side length $L$, and its plane wave factor $e^{i\left(y k_{y}+z k_{z}\right)}$ satisfies periodic boundary condition, i.e. $k_{y}=2 \pi n_{y} / L, k_{z}=2 \pi n_{z} / L,\left(n_{y}, n_{z} \in \mathbb{Z}\right)$. The center of the harmonic oscillator function $\varphi_{n}(\xi)$ is located at $x_{0}=k_{y} /(e B)$. The condition $0<x_{0}<L$ leads to $0<n_{y}<e B L^{2} /(2 \pi)$. Since the Landau levels do not depend on $k_{y}$, the degeneracy of each Landau level is $e B L^{2} /(2 \pi)$. The calculation details of Landau levels can be found in [13, 46, 47].

\section{GRAND PARTITION FUNCTION AND THERMODYNAMIC QUANTITIES}

Now we consider a system of massive Dirac fermions in a uniform magnetic field $\boldsymbol{B}=B \boldsymbol{e}_{z}$, where the interaction among fermions is ignored. This system is in equilibrium with a reservoir, which keeps constant temperature $T$ and constant chemical potential $\mu$. We can construct the grand partition function $\ln \Xi$ of this system from Landau levels in Sec. [I] as follows,

$$
\begin{aligned}
\ln \Xi= & \sum_{k_{y}, k_{z}}\left[\ln \left(1+e^{\beta \mu-\beta \sqrt{m^{2}+k_{z}^{2}}}\right)+\ln \left(1+e^{-\beta \mu-\beta \sqrt{m^{2}+k_{z}^{2}}}\right)\right] \\
& +2 \sum_{n=1}^{\infty} \sum_{k_{y}, k_{z}}\left[\ln \left(1+e^{\beta \mu-\beta \sqrt{2 n e B+m^{2}+k_{z}^{2}}}\right)+\ln \left(1+e^{-\beta \mu-\beta \sqrt{2 n e B+m^{2}+k_{z}^{2}}}\right)\right],
\end{aligned}
$$

where $\beta=1 / T$. We have ignored the vacuum term in $\ln \Xi$, which is equivalent to the normal ordering description of ensemble average approach [15, 43]. Here we are only interested in the medium induced correction, and neglect the vacuum contribution to the grand partition function. Actually the vacuum term would give an temperature-independent ultraviolet divergence which can be regularized by conventional re-normalization method at zero temperature. The summations over $k_{y}$ and $k_{z}$ in Eq. (8) can be replaced by the degeneracy factor $e B L^{2} /(2 \pi)$ and the integral $(L / 2 \pi) \int d k_{z}$ respectively.

The thermodynamic quantities of the system, such as particle number $N=V n$, energy $U=V \varepsilon$, pressure $p$, entropy $S=V s$ and magnetization intensity $M$, can be derived from $\ln \Xi$ in the following,

$$
N=\frac{\partial}{\partial a} \ln \Xi
$$




$$
\begin{gathered}
U=-\frac{\partial}{\partial \beta} \ln \Xi, \\
p=\frac{1}{\beta} \frac{\partial}{\partial V} \ln \Xi, \\
S=\ln \Xi+\beta U-a N, \\
M=\frac{1}{\beta} \frac{\partial}{\partial B}\left(\frac{\ln \Xi}{V}\right) .
\end{gathered}
$$

where $V=L^{3}$ is the volume of the system. After introducing an intensive quantity $g(a, b, c) \equiv\left(\beta^{3} \ln \Xi\right) / L^{3}$ and three dimensionless variables, $a=\beta \mu, b=2 e B \beta^{2}, c=\beta m$, the grand partition function in Eq. (8) becomes

$$
\begin{aligned}
g(a, b, c)= & \frac{b}{4 \pi^{2}} \int_{0}^{\infty} d s\left[\ln \left(1+e^{a-\sqrt{s^{2}+c^{2}}}\right)+\ln \left(1+e^{-a-\sqrt{s^{2}+c^{2}}}\right)\right] \\
& +\frac{b}{2 \pi^{2}} \int_{0}^{\infty} d s \sum_{n=1}^{\infty}\left[\ln \left(1+e^{a-\sqrt{n b+s^{2}+c^{2}}}\right)+\ln \left(1+e^{-a-\sqrt{n b+s^{2}+c^{2}}}\right)\right] .
\end{aligned}
$$

Now the extensive quantities in Eqs. $(9,13)$ becomes

$$
\begin{gathered}
n=\frac{1}{\beta^{3}} \frac{\partial}{\partial a} g(a, b, c), \\
\varepsilon=\frac{1}{\beta^{4}}\left(3-2 b \frac{\partial}{\partial b}-c \frac{\partial}{\partial c}\right) g(a, b, c), \\
p=\frac{1}{\beta^{4}} g(a, b, c), \\
s=\frac{1}{\beta^{3}}\left(4-a \frac{\partial}{\partial a}-2 b \frac{\partial}{\partial b}-c \frac{\partial}{\partial c}\right) g(a, b, c), \\
\chi=\frac{2 e}{\beta^{2}} \frac{\partial}{\partial b} g(a, b, c), \\
c_{T}=\frac{1}{\beta^{3}}\left(12-3 a \frac{\partial}{\partial a}-10 b \frac{\partial}{\partial b}-6 c \frac{\partial}{\partial c}+4 b^{2} \frac{\partial^{2}}{\partial b^{2}}+c^{2} \frac{\partial^{2}}{\partial c^{2}}\right. \\
\left.+2 a b \frac{\partial^{2}}{\partial a \partial b}+a c \frac{\partial^{2}}{\partial a \partial c}+4 b c \frac{\partial^{2}}{\partial b \partial c}\right) g(a, b, c),
\end{gathered}
$$

where $n, \varepsilon, p, s, M, \chi \equiv \partial M / \partial B, c_{T} \equiv \partial \varepsilon / \partial T$, are particle number density, energy density, pressure, entropy density, magnetization intensity, magnetic susceptibility and heat capacity, respectively. 


\section{EXPANSIONS OF INTENSIVE QUANTITIES AT $B=0$}

In order to study the analytic behaviors of all thermodynamic quantities at zero magnetic field, in this section all thermodynamic quantities will be expanded at $B=0$, i.e. at $b=0$ with $b=2 e B \beta^{2}$.

After defining an auxiliary function $f(a, x)$

$$
f(a, x)=\ln \left(1+e^{a-x}\right)+\ln \left(1+e^{-a-x}\right),
$$

$g(a, b, c)$ in Eq. (14) becomes

$$
g(a, b, c)=\frac{b}{2 \pi^{2}} \int_{0}^{\infty} d s\left[\frac{1}{2} f\left(a, \sqrt{s^{2}+c^{2}}\right)+\sum_{n=1}^{\infty} f\left(a, \sqrt{n b+s^{2}+c^{2}}\right)\right] .
$$

Making use of following Abel-Plana formula [48, 49]

$$
\frac{1}{2} \mathcal{F}(0)+\sum_{n=1}^{\infty} \mathcal{F}(n)=\int_{0}^{\infty} d t \mathcal{F}(t)+i \int_{0}^{\infty} d t \frac{\mathcal{F}(i t)-\mathcal{F}(-i t)}{e^{2 \pi t}-1}
$$

the summation over Landau levels in Eq. (23) can be transformed into integrations, i.e.

$$
\begin{aligned}
g(a, b, c)= & \frac{1}{2 \pi^{2}} \int_{0}^{\infty} d s \int_{0}^{\infty} d t f\left(a, \sqrt{t+s^{2}+c^{2}}\right) \\
& +\frac{b}{2 \pi^{2}} \times i \int_{0}^{\infty} d s \int_{0}^{\infty} d t \frac{f\left(a, \sqrt{i t b+s^{2}+c^{2}}\right)-f\left(a, \sqrt{-i t b+s^{2}+c^{2}}\right)}{e^{2 \pi t}-1},
\end{aligned}
$$

In Appendix A, we have expanded $g(a, b, c)$ at $b=0$ as follows,

$$
g(a, b, c)=\frac{1}{2 \pi^{2}} \int_{0}^{\infty} d s \int_{0}^{\infty} d t f\left(a, \sqrt{t+s^{2}+c^{2}}\right)-\frac{1}{\pi^{2}} \sum_{n=0}^{\infty} \frac{(4 n+1) ! !}{(4 n+4) ! !} \mathscr{B}_{2 n+2} C_{2 n+1}(a, c) b^{2 n+2}
$$

where $\mathscr{B}_{n}$ are Bernoulli numbers, and $C_{2 n+1}(a, c)(n \geqslant 0)$ is

$$
C_{2 n+1}(a, c)=-\frac{1}{(4 n+1) !} \int_{0}^{\infty} d y \ln y \frac{d^{4 n+2}}{d y^{4 n+2}} f\left(a, \sqrt{y^{2}+c^{2}}\right) .
$$

We can see that $g(a, b, c)$ in Eq. (26) is a power series of $b^{2}$.

Making use of the power series expansion of $g(a, b, c)$, the intensive quantities in Eqs. (15)-(21) become

$$
\begin{aligned}
n \beta^{3}= & \frac{1}{2 \pi^{2}} \int_{0}^{\infty} d s \int_{0}^{\infty} d t \frac{\partial}{\partial a} f\left(a, \sqrt{t+s^{2}+c^{2}}\right) \\
& -\frac{1}{\pi^{2}} \sum_{n=0}^{\infty} \frac{(4 n+1) ! !}{(4 n+4) ! !} \mathscr{B}_{2 n+2} \frac{\partial}{\partial a} C_{2 n+1}(a, c) b^{2 n+2},
\end{aligned}
$$




$$
\begin{aligned}
& \varepsilon \beta^{4}=\frac{1}{2 \pi^{2}} \int_{0}^{\infty} d s \int_{0}^{\infty} d t\left(3-c \frac{\partial}{\partial c}\right) f\left(a, \sqrt{t+s^{2}+c^{2}}\right) \\
& +\frac{1}{\pi^{2}} \sum_{n=0}^{\infty} \frac{(4 n+1) ! !}{(4 n+4) ! !} \mathscr{B}_{2 n+2}\left(4 n+1+c \frac{\partial}{\partial c}\right) C_{2 n+1}(a, c) b^{2 n+2} \\
& p \beta^{4}=\frac{1}{2 \pi^{2}} \int_{0}^{\infty} d s \int_{0}^{\infty} d t f\left(a, \sqrt{t+s^{2}+c^{2}}\right) \\
& -\frac{1}{\pi^{2}} \sum_{n=0}^{\infty} \frac{(4 n+1) ! !}{(4 n+4) ! !} \mathscr{B}_{2 n+2} C_{2 n+1}(a, c) b^{2 n+2} \\
& s \beta^{3}=\frac{1}{2 \pi^{2}} \int_{0}^{\infty} d s \int_{0}^{\infty} d t\left(4-a \frac{\partial}{\partial a}-c \frac{\partial}{\partial c}\right) f\left(a, \sqrt{t+s^{2}+c^{2}}\right) \\
& +\frac{1}{\pi^{2}} \sum_{n=0}^{\infty} \frac{(4 n+1) ! !}{(4 n+4) ! !} \mathscr{B}_{2 n+2}\left(4 n+a \frac{\partial}{\partial a}+c \frac{\partial}{\partial c}\right) C_{2 n+1}(a, c) b^{2 n+2} \\
& M \beta^{2} / e=-\frac{1}{\pi^{2}} \sum_{n=0}^{\infty} \frac{(4 n+1) ! !}{(4 n+2) ! !} \mathscr{B}_{2 n+2} C_{2 n+1}(a, c) b^{2 n+1}, \\
& \chi / e^{2}=-\frac{1}{\pi^{2}} \sum_{n=0}^{\infty} \frac{(4 n+1) ! !}{(4 n) ! !} \mathscr{B}_{2 n+2} C_{2 n+1}(a, c) b^{2 n}, \\
& c_{T} \beta^{3}=\frac{1}{2 \pi^{2}} \int_{0}^{\infty} d s \int_{0}^{\infty} d t\left(12-3 a \frac{\partial}{\partial a}-6 c \frac{\partial}{\partial c}+a c \frac{\partial^{2}}{\partial a \partial c}+c^{2} \frac{\partial^{2}}{\partial c^{2}}\right) f\left(a, \sqrt{t+s^{2}+c^{2}}\right) \\
& -\frac{1}{\pi^{2}} \sum_{n=0}^{\infty} \frac{(4 n+1) ! !}{(4 n+4) ! !} \mathscr{B}_{2 n+2}\left[4 n(4 n+1)+(4 n+1) a \frac{\partial}{\partial a}+(8 n+2) c \frac{\partial}{\partial c}\right. \\
& \left.+a c \frac{\partial^{2}}{\partial a \partial c}+c^{2} \frac{\partial^{2}}{\partial c^{2}}\right] C_{2 n+1}(a, c) b^{2 n+2},
\end{aligned}
$$

where we have used the temperature factor $\beta=1 / T$ and the electric charge $e$ to make all intensive quantities dimensionless. Now all intensive quantities are expanded at $b=0$ as a power series of $b$, i.e. they are all analytic at $b=0$. However, for the system of massless fermions as discussed in [45], only particle number density, entropy density and heat capacity are analytic at $b=0$, meanwhile, other intensive quantities such as energy density, pressure, magnetization intensity and magnetic susceptibility include a logarithmic term $\ln b$, which are not analytic at $b=0$.

According to the asymptotic behaviors of $C_{2 n+1}(a, c)(n \geqslant 0)$ when $c \rightarrow 0$, as discussed in Appendix B, the series expansions of $n, s$ and $c_{T}$ in Eqs. 28, 31, 34 can return to the results of the massless case [45], which is reasonable since these intensive quantities are analytic at $b=0$ for both massive and massless cases. 


\section{ZERO-FIELD MAGNETIC SUSCEPTIBILITY}

In this section, we investigate the magnetic susceptibility $\chi$ of the system at $B=0$, which is called zero-field magnetic susceptibility, denoted as $\chi_{0}$. According to Eq. (33), zero-field magnetic susceptibility can be expressed as

$$
\chi_{0}(a, c)=\frac{e^{2}}{6 \pi^{2}} \int_{0}^{\infty} d y \frac{1}{\sqrt{y^{2}+c^{2}}}\left(\frac{1}{e^{\sqrt{y^{2}+c^{2}}-a}+1}+\frac{1}{e^{\sqrt{y^{2}+c^{2}}+a}+1}\right) .
$$

We can see that the system of massive Dirac fermions is a paramagnetic system since $\chi_{0}>0$. In Figure 1. we plot the curves of $\chi_{0}$ with respect to the mass parameter $c=m / T$ at $a=\mu / T=0,1,2,3$. We find that $\chi_{0}$ is divergent at zero mass and decreases rapidly to zero at large mass, and larger chemical potential leads to larger $\chi_{0}$.

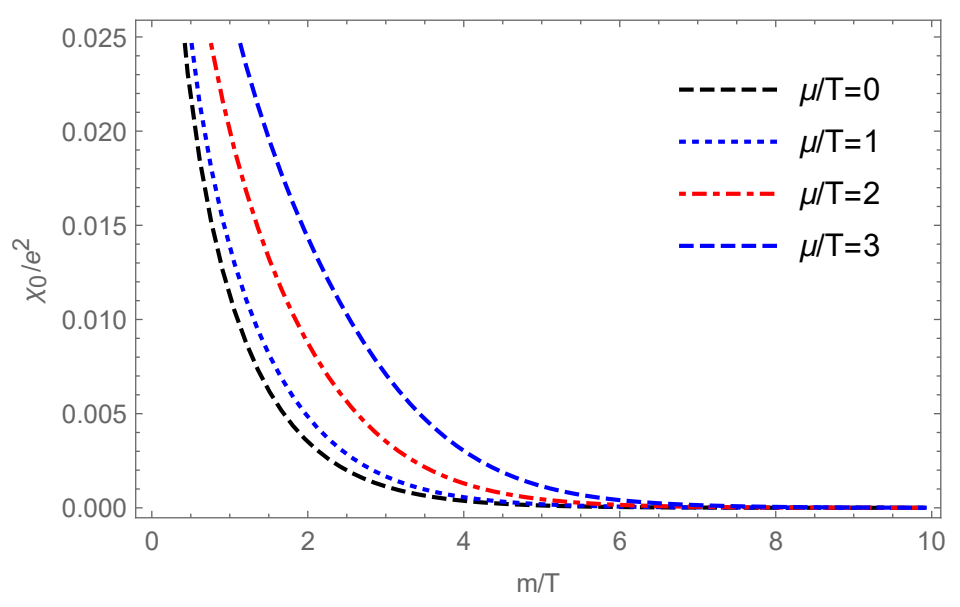

Figure 1. (color online) Mass dependence of $\chi_{0}$ at $\mu / T=0,1,2,3$.

In Appendix C, we obtained the expansion of $C_{1}(a, c)$ at $c=0$, so $\chi_{0}(a, c)$ can be expanded at $c=0$ as

$$
\chi_{0}(a, c)=\frac{e^{2}}{6 \pi^{2}}\left[-\frac{1}{2} \ln c^{2}+\left(D_{0}(a)+\ln 2\right)+\sum_{n=1}^{\infty} \frac{(2 n-1) ! !}{(2 n) ! !} D_{n}(a) c^{2 n}\right],
$$

where $D_{n}(a)(n \geqslant 0)$ is defined as

$$
D_{n}(a)=-\frac{1}{(2 n) !} \int_{0}^{\infty} d x \ln x \frac{d^{2 n+1}}{d x^{2 n+1}}\left(\frac{1}{e^{x-a}+1}+\frac{1}{e^{x+a}+1}\right) .
$$

This expansion for zero-field magnetic susceptibility is consistent with [11], where the imaginary time formalism and the proper time method is used. 
In the series expansion of $\chi_{0}(a, c)$ at $c=0$, besides the regular terms $c^{2 n}$, there is also a logarithmically divergent term $\ln c^{2}$ with a negative sign. In high temperature limit, the small mass parameter $c=m / T$ in the logarithmic term $\ln c^{2}$ will lead to a large value for $\chi_{0}$, i.e. the paramagnetic behavior of the system becomes stronger in higher temperature. This qualitative conclusion is consistent with recent lattice calculations [40, 42, where the authors considered the system of hot and dense QCD matter.

To obtain the asymptotic behavior of $\chi_{0}$ in large mass limit, another expression of Eq. (35) is useful,

$$
\chi_{0}=\frac{e^{2}}{6 \pi^{2}} \int_{c}^{\infty} \frac{d x}{\sqrt{x^{2}-c^{2}}}\left(\frac{1}{e^{x-a}+1}+\frac{1}{e^{x+a}+1}\right) .
$$

Suppose that $c \gg a>0$, then Eq. (38) becomes

$$
\frac{\chi_{0}}{e^{2}} \sim \frac{\cosh a}{3 \pi^{2}} \int_{c}^{\infty} \frac{d x}{\sqrt{x^{2}-c^{2}}} e^{-x}=\frac{\cosh a}{3 \pi^{2}} K_{0}(c) \sim \frac{\cosh a}{6 \pi \sqrt{c}} e^{-c},
$$

where $K_{0}(x)$ is the virtual Hankel function and we have used $\lim _{x \rightarrow \infty} K_{0}(x)=\frac{\pi}{2 \sqrt{x}} e^{-x}$. We find $\chi_{0}$ is exponentially suppressed at large mass. In low temperature limit, the large mass parameter $c=m / T$ will rapidly suppress the value of $\chi_{0}$ to be zero (always with a positive sign). However, in recent lattice calculation [40, the diamagnetic behavior (negative susceptibility) is found at low temperatures for QCD matter. The opposite sign of susceptibility in low temperature may results from the absence of interaction among particles in our calculation.

In nonrelativistic and strong degeneracy case, $\chi_{0}$ in Eq. 35 becomes

$$
\chi_{0}=\frac{e^{2}}{6 \pi^{2} c} \int_{0}^{\infty} d y \frac{1}{e^{\frac{y^{2}}{2 c}-\frac{\xi}{T}}+1}=-\frac{e^{2}}{6 \sqrt{2} \pi^{3 / 2} c^{1 / 2}} \operatorname{Li}_{1 / 2}\left(-e^{\xi / T}\right)
$$

where we have defined the nonrelativistic chemical potential $\xi \equiv T(a-c)$. Making use of $\lim _{x \rightarrow \infty} \operatorname{Li}_{1 / 2}\left(-e^{x}\right)=-\frac{\sqrt{x}}{\Gamma(3 / 2)}$, the strong degeneracy condition with $\xi / T \gg 1$ leads to

$$
\chi_{0}=\frac{e^{2}}{3 \sqrt{2} \pi^{2}} \sqrt{\frac{\xi}{m}}
$$

which indicates that the nonrelativistic electron gas with strong degeneracy displays weak paramagnetism [50]. In fact, $\chi_{0}$ in Eq. (41) can be decomposed into spin-related and orbital angular momentum-related contributions, $\chi_{0}^{\text {spin }}=\frac{3}{2} \chi_{0}, \chi_{0}^{\text {orbit }}=-\frac{1}{2} \chi_{0}$, which correspond to Pauli paramagnetism and Landau diamagnetism respectively [51, 52]. 


\section{SCALAR AND ENERGY-MOMENTUM TENSOR}

The macroscopic thermodynamic quantities of the system can be derived by the ensemble average of normal ordering of corresponding field operators. In this section we will calculate the scalar and the energy-momentum tensor of the system, which are defined as

$$
\begin{gathered}
F=\langle: \bar{\psi} \psi:\rangle, \\
T^{\mu \nu}=\frac{1}{4}\left\langle: \bar{\psi} \gamma^{\mu} i D^{\nu} \psi+\bar{\psi} \gamma^{\nu} i D^{\mu} \psi+\text { h.c. }:\right\rangle,
\end{gathered}
$$

where $\psi$ becomes a field operator, the angular brackets means statistical average, and the double dots enclosing the field operators means normal ordering as adopted in [15, 43, 45].

According to the rotation symmetry of the system along $z$-axis, the nonzero components of $T^{\mu \nu}$ are $T^{00}, T^{11}=T^{22}, T^{33}, T^{03}$. We have calculated energy density $\varepsilon=T^{00}$ in Eq. (29). In the following we will calculate $T^{11}=T^{22}, T^{33}, T^{03}$, and $F$.

The field operator $\psi(\boldsymbol{x})$ can be expanded by the orthonormal and complete eigenfunctions in Eqs. (3, 4) as follows,

$$
\begin{aligned}
\psi(\boldsymbol{x})= & \sum_{k_{y}, k_{z}}\left[a_{0}\left(k_{y}, k_{z}\right) \psi_{0+}\left(k_{y}, k_{z} ; \boldsymbol{x}\right)+b_{0}^{\dagger}\left(k_{y}, k_{z}\right) \psi_{0-}\left(k_{y}, k_{z} ; \boldsymbol{x}\right)\right] \\
& +\sum_{n, s, k_{y}, k_{z}}\left[a_{n s}\left(k_{y}, k_{z}\right) \psi_{n+s}\left(k_{y}, k_{z} ; \boldsymbol{x}\right)+b_{n s}^{\dagger}\left(k_{y}, k_{z}\right) \psi_{n-s}\left(k_{y}, k_{z} ; \boldsymbol{x}\right)\right]
\end{aligned}
$$

where $a_{n}, b_{n}, a_{n}^{\dagger}, b_{n}^{\dagger}$ are annihilation and creation operators for fermions and antifermions. As calculated in [43], the ensemble average of normal ordering of $a_{n}^{\dagger} a_{n}$ and $b_{n} b_{n}^{\dagger}$ are

$$
\begin{aligned}
\left\langle: a_{n s}^{\dagger}\left(k_{y}, k_{z}\right) a_{n s}\left(k_{y}, k_{z}\right):\right\rangle & =\frac{1}{e^{\beta\left(E_{n}-\mu\right)}+1}, \\
\left\langle: b_{n s}\left(k_{y}, k_{z}\right) b_{n s}^{\dagger}\left(k_{y}, k_{z}\right):\right\rangle & =-\frac{1}{e^{\beta\left(E_{n}+\mu\right)}+1} .
\end{aligned}
$$

Plugging Eq. (44) into Eqs. 42,43) and making use of Eqs. (45), (46), one can obtain $T^{03}=0$ and

$$
\begin{aligned}
T^{11} & =T^{22}=\frac{(e B)^{2}}{\pi^{2}} \sum_{n=1}^{\infty} \int_{0}^{\infty} d k_{z} \frac{n}{E_{n}}\left(\frac{1}{e^{\beta\left(E_{n}-\mu\right)}+1}+\frac{1}{e^{\beta\left(E_{n}+\mu\right)}+1}\right) \\
T^{33} & =\frac{e B}{\pi^{2}} \sum_{n=0}^{\infty} \frac{1}{1+\delta_{n, 0}} \int_{0}^{\infty} d k_{z} \frac{k_{z}^{2}}{E_{n}}\left(\frac{1}{e^{\beta\left(E_{n}-\mu\right)}+1}+\frac{1}{e^{\beta\left(E_{n}+\mu\right)}+1}\right) \\
F & =\frac{e B}{\pi^{2}} \sum_{n=0}^{\infty} \frac{1}{1+\delta_{n, 0}} \int_{0}^{\infty} d k_{z} \frac{m}{E_{n}}\left(\frac{1}{e^{\beta\left(E_{n}-\mu\right)}+1}+\frac{1}{e^{\beta\left(E_{n}+\mu\right)}+1}\right) .
\end{aligned}
$$


Due to the absence of axial chemical potential $\mu_{5}$ in our formulism, $T^{03}$ vanishes [16, 20]. Further calculations of $T^{11}, T^{22}, T^{33}$ and $F$ give

$$
\begin{aligned}
T^{11}=T^{22} & =\frac{1}{\beta^{4}}\left(1-b \frac{\partial}{\partial b}\right) g(a, b, c), \\
T^{33} & =\frac{1}{\beta^{4}} g(a, b, c)=p, \\
F & =-\frac{1}{\beta^{3}} \frac{\partial}{\partial c} g(a, b, c) .
\end{aligned}
$$

We find that $T^{33}$ is just the pressure $p$.

According to Dirac equation $\left(i \gamma^{\mu} D_{\mu}-m\right) \psi=0$, the trace of $T^{\mu \nu}$ is

$$
g_{\mu \nu} T^{\mu \nu}=T^{00}-T^{11}-T^{22}-T^{33}=m F
$$

The expressions of $T^{00}, T^{11}, T^{22}, T^{33}, F$ in Eqs. $16,50,52$ automatically satisfy Eq. (53). Now we calculate the massless limit of Eq. (53). From the series expansion of $g(a, b, c)$ and $F$ in Eqs. 26, 52, the trace equation becomes

$$
\begin{aligned}
g_{\mu \nu} T^{\mu \nu}= & \frac{c}{\beta^{4}}\left[-\frac{1}{2 \pi^{2}} \int_{0}^{\infty} d s \int_{0}^{\infty} d t \frac{\partial}{\partial c} f\left(a, \sqrt{t+s^{2}+c^{2}}\right)\right. \\
& \left.+\frac{1}{\pi^{2}} \sum_{n=0}^{\infty} \frac{(4 n+1) ! !}{(4 n+4) ! !} \mathscr{B}_{2 n+2} \frac{\partial}{\partial c} C_{2 n+1}(a, c) b^{2 n+2}\right] .
\end{aligned}
$$

The first integration in Eq. (54) tends to be zero as $c \rightarrow 0$. Making use of the asymptotic behaviors of $C_{n}(a, c)$ at $c=0$ in Appendix $\mathrm{B}$, one can obtain

$$
\lim _{c \rightarrow 0} c \frac{\partial}{\partial c} C_{n}(a, c)=\delta_{n, 1}
$$

Then Eq. (54) gives

$$
\lim _{c \rightarrow 0} g_{\mu \nu} T^{\mu \nu}=\frac{e^{2} B^{2}}{12 \pi^{2}},
$$

which is just the trace anomaly equation [20, 53] in the case of a pure magnetic field with the field strength invariant $F^{\mu \nu} F_{\mu \nu}=2 B^{2}$.

\section{MASS CORRECTION TO CHIRAL SEPARATION EFFECT}

Similar to the calculation for $T^{\mu \nu}$ in Sec. VI, the vector and axial currents $J_{V}^{\mu}, J_{A}^{\mu}$ can also be derived through ensemble average, i.e.

$$
J_{V}^{\mu}=\left\langle: \bar{\psi} \gamma^{\mu} \psi:\right\rangle,
$$




$$
J_{A}^{\mu}=\left\langle: \bar{\psi} \gamma^{\mu} \gamma^{5} \psi:\right\rangle .
$$

The zero component of $J_{V}^{\mu}$ is just the particle number density calculated in Eq. 28). Plugging Eq. (44) into Eqs. (57, 58) and making use of Eqs. (45, 46) one can obtain

$$
\begin{gathered}
J_{V}^{i}=J_{A}^{k}=J_{A}^{0}=0, \quad(i=1,2,3 ; k=1,2), \\
J_{A}^{3}=\frac{e B}{2 \pi^{2}} \int_{0}^{\infty} d k_{z}\left(\frac{1}{e^{\beta\left(\sqrt{m^{2}+k_{z}^{2}}-\mu\right)}+1}-\frac{1}{e^{\beta\left(\sqrt{m^{2}+k_{z}^{2}}+\mu\right)}+1}\right) .
\end{gathered}
$$

Due to the absence of axial chemical potential $\mu_{5}$ in our formulism, $J_{V}^{i}$ and $J_{A}^{0}$ vanish [13, 16, 20]. Fluctuation and dissipation of axial charge $J_{A}^{0}$ from massive quarks is discussed by one of the authors [54]. The nonzero component $J_{A}^{3}$ is linear for the magnetic field $B$, which is the chiral separation effect (CSE) for the system of massive Dirac fermions [35, 55].

If we take $m=0$, the coefficient of $e B$ in Eq. 60) can be integrated out,

$$
J_{A}^{3}=\frac{e B \mu}{2 \pi^{2}} .
$$

We know that, for massless fermion system, the dynamics of right- and left-handed fermions decouples, so we can introduce the particle number currents $J_{R}^{\mu}, J_{L}^{\mu}$ for right- and left-handed fermion systems, respectively. Now the vector and axial currents $J_{V}^{\mu}, J_{A}^{\mu}$ can be expressed as

$$
J_{V}^{\mu}=J_{R}^{\mu}+J_{L}^{\mu}, \quad J_{A}^{\mu}=J_{R}^{\mu}-J_{L}^{\mu} .
$$

Since we have set $\mu_{5}=0$, then chemical potentials $\mu_{R}, \mu_{L}$ of right- and left-handed fermions are equal. Combining Eq. (59) and Eq. (62), one can obtain

$$
J_{R}^{3}=\frac{e B \mu_{R}}{4 \pi^{2}}, \quad J_{L}^{3}=-\frac{e B \mu_{L}}{4 \pi^{2}},
$$

which are the chiral magnetic effects for right- and left-handed fermion systems respectively [26, 56].

We rewrite Eq. 60 as $J_{A}^{3}=B \sigma_{B}$, and $\sigma_{B}$ is the CSE coefficient,

$$
\sigma_{B}=\frac{e T}{2 \pi^{2}} \int_{0}^{\infty} d x\left(\frac{1}{e^{\sqrt{x^{2}+c^{2}}-a}+1}-\frac{1}{e^{\sqrt{x^{2}+c^{2}}+a}+1}\right),
$$

which is consistent with [35], where the authors showed that the presence of mass generically suppresses the CSE coefficient with less suppression at larger chemical potential. 
At zero temperature, Eq. (64) gives

$$
\sigma_{B}=\frac{e}{2 \pi^{2}} \sqrt{\mu^{2}-m^{2}}[\theta(\mu-m)-\theta(-\mu-m)]
$$

which vanishes for $\mu^{2}<m^{2}$, and returns to $\frac{e \mu}{2 \pi^{2}}$ in massless limit. This agrees with the free case [35, 55].

At nonzero temperature, we will expand the the CSE coefficient $\sigma_{B}$ at $m=0$. Another expression of Eq. (64) is useful,

$$
\begin{gathered}
\sigma_{B}=\frac{e T}{2 \pi^{2}} \frac{d}{d a} F(a, c), \\
F(a, c)=\int_{|c|}^{\infty} d y \frac{y}{\sqrt{y^{2}-c^{2}}}\left[\ln \left(1+e^{a-y}\right)+\ln \left(1+e^{-a-y}\right)\right] .
\end{gathered}
$$

In the recent article by some of us [45], Eqs. (B3, B12, B14) can give

$$
\sigma_{B}=\frac{e T}{2 \pi^{2}} \sum_{n=0}^{\infty} \frac{(2 n-1) ! !}{(2 n) ! !} E_{n}(a)\left(\frac{m}{T}\right)^{2 n}
$$

where $E_{n}(a)$ are defined as

$$
\begin{gathered}
E_{0}(a)=a, \\
E_{n}(a)=\frac{1}{(2 n-1) !} \frac{d^{2 n}}{d a^{2 n}}\left\{\frac{d}{d s}\left[\operatorname{Li}_{s}\left(-e^{a}\right)-\operatorname{Li}_{s}\left(-e^{-a}\right)\right]\right\}_{s=1}, \quad(n \geqslant 1),
\end{gathered}
$$

and $\operatorname{Li}_{s}(z)$ is the polylogarithm function. The $n=1$ term in Eq. (68) is consistent with [35]. There is no logarithmic term $\ln (m / T)$ in the expansion of $\sigma_{B}$, which is different from the case of zero-field magnetic susceptibility discussed in Sec. V. In the recent article [45], Eqs. (D9, C9) can give following asymptotic behaviors of $E_{n}(a)$ at small and lager chemical potential respectively,

$$
\begin{gathered}
\lim _{a \rightarrow 0} E_{n}(a)=(-1)^{n} \frac{2 n}{\pi^{2 n}} \zeta(2 n+1)\left(2-2^{-2 n}\right) a, \\
\lim _{a \rightarrow \infty} E_{n}(a)=-\frac{1}{2 n-1} \frac{1}{a^{2 n-1}} .
\end{gathered}
$$

Making use of $\lim _{s \rightarrow 0} s \zeta(s+1)=1$, we find that Eqs. 71, 72 both apply to $n=0$ case. The asymptotic behavior in Eq. (72) implies that mass corrections to $\sigma_{B}$ at high orders become smaller at larger chemical potential. 


\section{SUMMARY}

In this article we have studied the influence of the magnetic field on the system of massive Dirac fermions. From the Landau levels for a single massive Dirac fermion in a uniform magnetic field, we construct the partition function for the system, through which all thermodynamic intensive quantities can be derived. Making use of Abel-Plana formula, all thermodynamic quantities can be expanded as power series of the dimensionless variable $b=2 e B / T^{2}$, i.e. they are all analytic at $b=0$, which is different from the massless case. We expand the zero-field magnetic susceptibility $\chi_{0}$ at $m=0$, and a logarithmic term appears at leading order. The asymptotic behavior of $\chi_{0}$ at high temperature is qualitatively consistent with recent lattice calculations. In nonrelativistic limit, $\chi_{0}$ can return to the result of electron gas with strong degeneracy. The macroscopic thermodynamic quantities, such as scalar, vector current, axial vector current and energy-momentum tensor, can be obtained by the ensemble average of normal ordering of corresponding field operators. For massless chiral fermions, our results recover the chiral magnetic effect for right- and left-handed fermions. We discuss mass correction to chiral separation effect. The presence of mass exponentially suppresses the CSE coefficient, and we expand the CSE coefficient at $m=0$ to any order.

\section{ACKNOWLEDGMENTS}

We thank Hai-Cang Ren and Xin-Li Sheng for helpful discussions. This work is supported by the National Natural Science Foundation of China (Grant Nos. 11890713, 11735007, 11890711, and 11947228), and the Chinese Postdoctoral Science Foundation (Grant No. 2019M662316).

\section{Appendix A: Expansion of $g(a, b, c)$ at $b=0$}

Making use of Abel-Plana formula, the grand partition function can be expressed as

$g(a, b, c)=\frac{1}{2 \pi^{2}} \int_{0}^{\infty} d s \int_{0}^{\infty} d t f\left(a, \sqrt{t+s^{2}+c^{2}}\right)+\frac{b}{2 \pi^{2}} \times i \int_{0}^{\infty} d t \frac{F(a, c, \sqrt{i t b})-F(a, c, \sqrt{-i t b})}{e^{2 \pi t}-1}$.

where $a=\mu \beta, b=2 e B \beta^{2}, c=m \beta$ and $f(a, x), F(a, c, x)$ are defined as

$$
f(a, x)=\ln \left(1+e^{a-x}\right)+\ln \left(1+e^{-a-x}\right),
$$




$$
F(a, c, x)=\int_{0}^{\infty} d s f\left(a, \sqrt{x^{2}+s^{2}+c^{2}}\right) .
$$

By the variable transformation $y=\sqrt{x^{2}+s^{2}}$ in Eq. A3), $F(a, c, x)$ can be rewritten as

$$
F(a, c, x)=\int_{|x|}^{\infty} d y \frac{y}{\sqrt{y^{2}-x^{2}}} f\left(a, \sqrt{y^{2}+c^{2}}\right) .
$$

Making use of following Taylor expansion,

$$
\frac{y}{\sqrt{y^{2}-x^{2}}}=\sum_{n=0}^{\infty} \frac{(2 n-1) ! !}{(2 n) ! !} \frac{x^{2 n}}{y^{2 n}}
$$

$F(a, c, x)$ becomes

$$
F(a, c, x)=\sum_{n=0}^{\infty} \frac{(2 n-1) ! !}{(2 n) ! !} x^{2 n} d_{n}(a, c, x),
$$

where we have defined $d_{n}(a, c, x)$ as

$$
d_{n}(a, c, x)=\int_{|x|}^{\infty} d y \frac{1}{y^{2 n}} f\left(a, \sqrt{y^{2}+c^{2}}\right) .
$$

Since $d_{n}(a, c, x)=d_{n}(a, c,-x)$, the derivative of $d_{n}(a, c, x)$ with respect to $x$ is

$$
d_{n}^{\prime}(a, c, x)=-\frac{|x|}{x^{2 n+1}} f\left(a, \sqrt{x^{2}+c^{2}}\right) .
$$

We can expand $f\left(a, \sqrt{x^{2}+c^{2}}\right)$ at $x=0$ as follows,

$$
f\left(a, \sqrt{x^{2}+c^{2}}\right)=\sum_{k=0}^{\infty} w_{2 k}(a, c) x^{2 k}
$$

then $d_{n}(a, c, x)$ becomes

$$
d_{n}(a, c, x)=|x| \sum_{k=0}^{\infty} w_{2 k}(a, c) \frac{1}{2 n-2 k-1} x^{2 k-2 n}+C_{n}(a, c),
$$

where $C_{n}(a, c)$ is independent of $x$. Now $F(a, c, x)$ in Eq. A6 becomes

$$
F(a, c, x)=\sum_{n=0}^{\infty} \frac{(2 n-1) ! !}{(2 n) ! !} C_{n}(a, c) x^{2 n}
$$

where we have used

$$
\sum_{n=0}^{\infty} \frac{(2 n-1) ! !}{(2 n) ! !} \frac{1}{2 n-2 k-1}=0, \quad(k=0,1,2, \cdots)
$$


The coefficient $C_{n}(a, c)$ in Eq. A11) is the constant term when $d_{n}(a, c, x)$ in expanded at $x=0$. When $n>0$, we can rewrite $d_{n}(a, c, x)$ in (A7) through integration by parts as

$$
\begin{aligned}
d_{n}(a, c, x)= & \sum_{k=0}^{2 n-2} \frac{(2 n-k-2) !}{(2 n-1) !} \frac{1}{x^{2 n-k-1}} \frac{d^{k}}{d x^{k}} f\left(a, \sqrt{x^{2}+c^{2}}\right)-\frac{\ln x}{(2 n-1) !} \frac{d^{2 n-1}}{d x^{2 n-1}} f\left(a, \sqrt{x^{2}+c^{2}}\right) \\
& -\frac{1}{(2 n-1) !} \int_{x}^{\infty} d y \ln y \frac{d^{2 n}}{d y^{2 n}} f\left(a, \sqrt{y^{2}+c^{2}}\right) .
\end{aligned}
$$

which implies

$$
C_{n}(a, c)=-\frac{1}{(2 n-1) !} \int_{0}^{\infty} d y \ln y \frac{d^{2 n}}{d y^{2 n}} f\left(a, \sqrt{y^{2}+c^{2}}\right) .
$$

When $n=0$, we have

$$
C_{0}(a, c)=\int_{0}^{\infty} d y f\left(a, \sqrt{y^{2}+c^{2}}\right)
$$

Substituting Eq. A11 into Eq. A1 gives

$$
g(a, b, c)=\frac{1}{2 \pi^{2}} \int_{0}^{\infty} d s \int_{0}^{\infty} d t f\left(a, \sqrt{t+s^{2}+c^{2}}\right)-\frac{1}{\pi^{2}} \sum_{n=0}^{\infty} \frac{(4 n+1) ! !}{(4 n+4) ! !} \mathscr{B}_{2 n+2} C_{2 n+1}(a, c) b^{2 n+2}
$$

where we have used following integrations,

$$
\int_{0}^{\infty} d t \frac{t^{2 n+1}}{e^{2 \pi t}-1}=(-1)^{n} \frac{\mathscr{B}_{2 n+2}}{4 n+4}, \quad(n \geqslant 0),
$$

with Bernoulli numbers $\mathscr{B}_{n}$ defined as

$$
\frac{t}{e^{t}-1}=\sum_{n=0}^{\infty} \frac{\mathscr{B}_{n}}{n !} t^{n}
$$

Appendix B: Asymptotic behaviors of $C_{n}(a, c)(n \geqslant 1)$ at $c=0$

The expression of $C_{n}(a, c)(n \geqslant 1)$ in Eq. (A14) is

$$
C_{n}(a, c)=-\frac{1}{(2 n-1) !} \int_{0}^{\infty} d y \ln y \frac{d^{2 n}}{d y^{2 n}} f\left(a, \sqrt{y^{2}+c^{2}}\right)
$$

where $f(a, x)=\ln \left(1+e^{a-x}\right)+\ln \left(1+e^{-a-x}\right)$. Making use of integration by parts, Eq. (B1) becomes

$$
C_{n}(a, c)=-\frac{1}{(2 n-1) !} \int_{0}^{\infty} d y \frac{1}{y} \frac{d^{2 n-2}}{d y^{2 n-2}}\left[\left(\frac{1}{e^{\sqrt{y^{2}+c^{2}}-a}+1}+\frac{1}{e^{\sqrt{y^{2}+c^{2}}+a}+1}\right) \frac{y}{\sqrt{y^{2}+c^{2}}}\right],
$$


where the second equal sign is valid for $n>1$ with an arbitrary $c$, and for $n=1$ with $c \neq 0$.

When $c=0$, Eq. (B2) becomes

$$
C_{n}(a, 0)=-\frac{1}{(2 n-1) !} \int_{0}^{\infty} d y \frac{1}{y} \frac{d^{2 n-2}}{d y^{2 n-2}}\left(\frac{1}{e^{y-a}+1}+\frac{1}{e^{y+a}+1}\right) .
$$

The expression in the bracket in the integrand can be expanded at $y=0$ as follows,

$$
\frac{1}{e^{y-a}+1}+\frac{1}{e^{y+a}+1}=1+\# y+\# y^{3}+\# y^{5}+\cdots,
$$

with "\#" representing some coefficients independent of $y$. The expansion in Eq. (B4) implies that, $C_{n}(a, 0)$ is divergent for $n=1$, and convergent for $n>1$. In Sec. C, we have proved that, the asymptotic behavior of $C_{1}(a, c)$ at $c=0$ is

$$
C_{1}(a, c)=\frac{1}{2} \ln c^{2}+\left(c^{2 k} \text { terms with } k \geqslant 0\right) .
$$

Now we analyse the asymptotic behaviors of $C_{n}(a, c)$ at $c=0$ for $n>1$. The derivative of $C_{n}(a, c)$ with respect to $c^{2}$ is

$$
\frac{d}{d c^{2}} C_{n}(a, c)=\frac{1}{(2 n-1) !} \int_{0}^{\infty} d y \ln y \frac{d^{2 n}}{d y^{2 n}}\left[\frac{1}{2 \sqrt{y^{2}+c^{2}}}\left(\frac{1}{e^{\sqrt{y^{2}+c^{2}}-a}+1}+\frac{1}{e^{\sqrt{y^{2}+c^{2}}+a}+1}\right)\right] .
$$

When $c=0$, the asymptotic behavior of the expression in the square brackets in Eq. (B6) at $y=0$ is $1 / y$, then the integration in Eq. (B6) is divergent. Since $C_{n}(a, 0)$ is finite, we can obtain

$$
C_{n}(a, c)=\# c^{2} \ln c^{2}+\left(c^{2 k} \text { terms with } k \geqslant 0\right) .
$$

\section{Appendix C: Expansion of $C_{1}(a, c)$ at $c=0$}

When $n=1$, Eq. (B2) becomes

$$
\begin{aligned}
C_{1}(a, c) & =-\int_{0}^{\infty} d y \frac{1}{\sqrt{y^{2}+c^{2}}}\left(\frac{1}{e^{\sqrt{y^{2}+c^{2}}-a}+1}+\frac{1}{e^{\sqrt{y^{2}+c^{2}}+a}+1}\right) \\
& =-\int_{|c|}^{\infty} d x \frac{1}{x}\left(1-\frac{c^{2}}{x^{2}}\right)^{-\frac{1}{2}}\left(\frac{1}{e^{x-a}+1}+\frac{1}{e^{x+a}+1}\right) .
\end{aligned}
$$

Since $x^{2}>c^{2}$, we can use the Taylor expansion for $\left(1-c^{2} / x^{2}\right)^{-1 / 2}$ in Eq. A5, which leads to

$$
C_{1}(a, c)=-\sum_{n=0}^{\infty} \frac{(2 n-1) ! !}{(2 n) ! !} c^{2 n} X_{n}(a, c)
$$


where $X_{n}(a, c)$ can be written as follows,

$$
X_{n}(a, c)=\int_{|c|}^{\infty} d x\left[\frac{1}{x^{2 n+1}}\left(\frac{1}{e^{x-a}+1}+\frac{1}{e^{x+a}+1}-1\right)+\frac{1}{x^{2 n+1}}\right] .
$$

The derivative of $X_{n}(a, c)$ with respect to $c$ is

$$
X_{n}^{\prime}(a, c)=-\frac{|c|}{c^{2 n+2}}\left(\frac{1}{e^{c-a}+1}+\frac{1}{e^{c+a}+1}-1\right)-\frac{1}{c^{2 n+1}} .
$$

The term in the bracket in Eq. (C4) is an odd function of $c$ which can be expanded at $c=0$ as

$$
\frac{1}{e^{c-a}+1}+\frac{1}{e^{c+a}+1}-1=\sum_{k=0}^{\infty} v_{2 k+1}(a) c^{2 k+1},
$$

then $X_{n}(a, c)$ can be obtained from $X_{n}^{\prime}(a, c)$,

$$
X_{n}(a, c)=-|c| \sum_{k=0}^{\infty} v_{2 k+1}(a) \frac{1}{2 k-2 n+1} c^{2 k-2 n}+\left\{\begin{array}{c}
D_{0}(a)-\frac{1}{2} \ln c^{2}, \quad n=0 \\
D_{n}(a)+\frac{1}{2 n} c^{-2 n}, n>0
\end{array}\right.
$$

where $D_{n}(a)$ are independent of $c$ and can be determined by the same method as the calculation of $C_{n}(a, c)$ in Sec. $\mathrm{A}$. The result of $D_{n}(a)$ is

$$
D_{n}(a)=-\frac{1}{(2 n) !} \int_{0}^{\infty} d x \ln x \frac{d^{2 n+1}}{d x^{2 n+1}}\left(\frac{1}{e^{x-a}+1}+\frac{1}{e^{x+a}+1}\right) .
$$

Making use of

$$
\begin{aligned}
\sum_{n=0}^{\infty} \frac{(2 n-1) ! !}{(2 n) ! !} & \frac{1}{2 n-2 k-1}=0, \quad(k=0,1,2, \cdots) \\
& \sum_{n=1}^{\infty} \frac{(2 n-1) ! !}{(2 n) ! !} \frac{1}{n}=\ln 4
\end{aligned}
$$

we get

$$
C_{1}(a, c)=\frac{1}{2} \ln c^{2}-\left[D_{0}(a)+\ln 2\right]-\sum_{n=1}^{\infty} \frac{(2 n-1) ! !}{(2 n) ! !} D_{n}(a) c^{2 n} .
$$

According to the Appendix D in [45], $D_{n}(a)$ can be expanded at $a=0$ as follows,

$$
D_{n}(a)=(-\ln 4-\gamma) \delta_{n, 0}-\frac{2}{(2 n) !} \sum_{k=0}^{\infty}\left(2^{2 n+2 k+1}-1\right) \zeta^{\prime}(-2 n-2 k) \frac{a^{2 k}}{(2 k) !}
$$

[1] P. A. Dirac, Proc. Roy. Soc. Lond. A 117, 610 (1928). 
[2] C. Anderson, Phys. Rev. 43, 491 (1933).

[3] W. Heisenberg and H. Euler, Z. Phys. 98, 714 (1936), physics/0605038.

[4] V. Weisskopf, Kong. Dan. Vid. Sel. Mat. Fys. Med. 14N6, 1 (1936).

[5] J. Schwinger, Phys. Rev. 82, 664 (1951), URL https://link.aps.org/doi/10.1103/ PhysRev.82.664.

[6] A. Salam and J. Strathdee, Nucl. Phys. B 90, 203 (1975).

[7] S. K. Blau, M. Visser, and A. Wipf, Int. J. Mod. Phys. A 6, 5409 (1991), 0906.2851.

[8] L. Dolan and R. Jackiw, Phys. Rev. D 9, 3320 (1974).

[9] E. V. Shuryak, Phys. Rept. 61, 71 (1980).

[10] J. Kapusta and C. Gale, Finite-temperature field theory: principles and applications (2nd edition), Cambridge university press, Cambridge (2006).

[11] D. Cangemi and G. V. Dunne, Annals Phys. 249, 582 (1996), hep-th/9601048.

[12] F. Hebenstreit, R. Alkofer, and H. Gies, Phys. Rev. D 82, 105026 (2010), 1007.1099.

[13] X.-l. Sheng, D. H. Rischke, D. Vasak, and Q. Wang, Eur. Phys. J. A54, 21 (2018), 1707.01388.

[14] X.-L. Sheng, R.-H. Fang, Q. Wang, and D. H. Rischke, Phys. Rev. D99, 056004 (2019), 1812.01146 .

[15] D. Vasak, M. Gyulassy, and H. T. Elze, Annals Phys. 173, 462 (1987).

[16] J.-H. Gao, Z.-T. Liang, S. Pu, Q. Wang, and X.-N. Wang, Phys. Rev. Lett. 109, 232301 (2012), 1203.0725 .

[17] J.-W. Chen, S. Pu, Q. Wang, and X.-N. Wang, Phys. Rev. Lett. 110, 262301 (2013), 1210.8312.

[18] Y. Hidaka, S. Pu, and D.-L. Yang, Phys. Rev. D95, 091901 (2017), 1612.04630.

[19] J.-H. Gao, Z.-T. Liang, Q. Wang, and X.-N. Wang, Phys. Rev. D98, 036019 (2018), 1802.06216.

[20] S.-Z. Yang, J.-H. Gao, Z.-T. Liang, and Q. Wang, Phys. Rev. D 102, 116024 (2020), 2003.04517 .

[21] X. Guo, Chin. Phys. C 44, 104106 (2020), 2005.00228.

[22] K. Itokazu, K. Yanase, and N. Yoshinaga, JPS Conf. Proc. 23, 013003 (2018).

[23] A. Reisenegger (2013), 1305.2542.

[24] S. Islam and S. Basu, Chin. Phys. Lett. 35, 099501 (2018), 1812.02568.

[25] D. E. Kharzeev, L. D. McLerran, and H. J. Warringa, Nucl. Phys. A803, 227 (2008), 0711.0950.

[26] K. Fukushima, D. E. Kharzeev, and H. J. Warringa, Phys. Rev. D78, 074033 (2008), 0808.3382.

[27] B. Feng, D.-F. Hou, and H.-C. Ren, Phys. Rev. D 99, 036010 (2019), 1810.05954. 
[28] S. Shi, J. Liao, and M. Gyulassy, Chin. Phys. C 43, 044101 (2019), 1808.05461.

[29] J. Adam et al. (STAR), Nucl. Sci. Tech. 32, 48 (2021), 1911.00596.

[30] G.-R. Liang, J. Liao, S. Lin, L. Yan, and M. Li, Chin. Phys. C 44, 094103 (2020), 2004.04440.

[31] J.-H. Gao, G.-L. Ma, S. Pu, and Q. Wang, Nucl. Sci. Tech. 31, 90 (2020), 2005.10432.

[32] Y.-C. Liu and X.-G. Huang, Nucl. Sci. Tech. 31, 56 (2020), 2003.12482.

[33] D. T. Son and A. R. Zhitnitsky, Phys. Rev. D 70, 074018 (2004), hep-ph/0405216.

[34] M. A. Metlitski and A. R. Zhitnitsky, Phys. Rev. D 72, 045011 (2005), hep-ph/0505072.

[35] S. Lin and L. Yang, Phys. Rev. D 98, 114022 (2018), 1810.02979.

[36] G. Bali, F. Bruckmann, G. Endrodi, F. Gruber, and A. Schaefer, JHEP 04, 130 (2013), 1303.1328.

[37] S. Mao, Chin. Phys. Lett. 33, 112501 (2016), 1509.05151.

[38] S. Mao, Y. Wu, and P. Zhuang, JPS Conf. Proc. 20, 011009 (2018).

[39] A. Ballon-Bayona, J. P. Shock, and D. Zoakos, JHEP 10, 193 (2020), 2005.00500.

[40] G. S. Bali, G. Endrődi, and S. Piemonte, JHEP 07, 183 (2020), 2004.08778.

[41] H. T. Ding, S. T. Li, Q. Shi, A. Tomiya, X. D. Wang, and Y. Zhang, Acta Phys. Polon. Supp. 14, 403 (2021), 2011.04870.

[42] P. V. Buividovich, D. Smith, and L. Von Smekal (2021), 2104.10012.

[43] R.-D. Dong, R.-H. Fang, D.-F. Hou, and D. She, Chin. Phys. C 44, 074106 (2020), 2001.05801.

[44] J.-H. Gao, Z.-T. Liang, and Q. Wang, Phys. Rev. D 101, 096015 (2020), 1910.11060.

[45] C. Zhang, R.-H. Fang, J.-H. Gao, and D.-F. Hou, Phys. Rev. D 102, 056004 (2020), 2005.08512.

[46] M.-A. Dariescu and C. Dariescu, Chin. Phys. Lett. 32, 071101 (2015).

[47] X.-L. Sheng, Ph.D. thesis, Frankfurt U. (2019), 1912.01169.

[48] G.-J. Ni and S.-Q. Chen, Advanced quantum mechanics (2nd edition), Fudan University Press, Shanghai (2003).

[49] P. L. Butzer, P. J. S. G. Ferreira, G. Schmeisser, and R. L. Stens, Results in mathematics, 59(3): 359-400 (2011).

[50] H.-W. Peng and X.-S. Xu, Foundations of theoretical physics, Peking university Press, Beijing (2011).

[51] W. Pauli, Z. Phys. 41, 81 (1927).

[52] L. Landau, Z. Phys. 64, 629 (1930).

[53] M. Peskin and D. Schroeder, An introduction to quantum field theory, Westview Press, New 
York (1995).

[54] D.-f. Hou and S. Lin, Phys. Rev. D 98, 054014 (2018), 1712.08429.

[55] E. V. Gorbar, V. A. Miransky, I. A. Shovkovy, and X. Wang, Phys. Rev. D 88, 025025 (2013), 1304.4606.

[56] D. E. Kharzeev and D. T. Son, Phys. Rev. Lett. 106, 062301 (2011), 1010.0038. 